\section{Masern-Endemie in Deutschland}

Die WHO möchte die Masern ausrotten. Um dieses Ziel zu erreichen, müssten 95\% der Kinder zwei Dosen einer MMR-Vakzine erhalten. In Deutschland liegt die Durchimpfungsrate bei 89\%. Aber selbst in Regionen mit überdurchschnittlichen Durchimpfungsraten kann es noch zu Masernendemien kommen, wie das Beispiel Essen (Durchimpfungsrate 92\%) zeigt.

D eutsche Wissenschaftler beschrieben fünf Masernfälle bei Schulkindern, die alle in eine Waldorfschule in Essen gingen. Die Erkrankungen wurden am 15. März 2010 diagnostiziert. Vier Tage später wurden bereits 71 Masernfälle (68 Kinder und drei Erwachsene) registriert. Davon waren

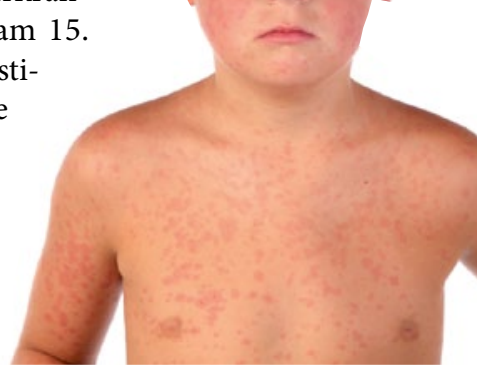

30 Waldorfschulkinder oder deren Geschwister. Von den 71 Fällen hatte nur ein Kind zwei MMR-Impfungen erhalten.

Serologisch war das Virus dem sehr ähnlich, das bereits im selben Jahr in einer Ber-

liner Waldorfschule $\mathrm{zu}$ einer Masernendemie geführt hatte. Dort war es zu 62 dokumentierten Erkrankungsfällen gekommen.

\title{
Kann man gegen Erdnuss oral hyposensibilisieren?
}

Über erste Erfahrungen mit einer oralen Immuntherapie bei Erdnussallergie berichtete jetzt eine Arbeitsgruppe um Katharina Blümchen aus der Berliner Charité.

inderärzte aus Berlin und New York untersuchten 23 Kinder zwischen drei und 14 Jahren, die eine Erdnussallergie im doppelblinden und placebokontrollierten Provokationstest (DBPCFC) und im IgE-RAST hatten. Die Hyposensibilisierung in Form von gerösteten Erdnüssen wurde mit einem siebentägigen Rush-Protokoll eingeleitet, bei Nichterreichen der protektiven Dosis von mindestens 0,5 g erhielten die Kinder eine Langzeit-Titration mit Dosissteigerungen alle zwei Wochen. Die Erhaltungsphase betrug acht Wochen und nach zweiwöchiger Karenz wurden die Patienten nochmals per DBPCFC provoziert.

Im Ergebnis tolerierten die Kinder nach der Rush-Aufdosierung über sieben Tage im Median lediglich eine Dosis von 0,15 g Erdnuss. 22 der 23 Patienten bekamen daher die Langzeitaufdosierung. Nach sieben Monaten erreichten 14 Patienten die anvisierte Schutzdosis von 0,5 g Erdnuss, im abschließenden DBPCFC wurde im Median 1 g (0,25-4 g) vertragen, vor der oralen Immuntherapie waren es nur $0,19 \mathrm{~g}(0,02-1 \mathrm{~g})$.

Die Nebenwirkungen waren erträglich: bei $2,6 \%$ von insgesamt 6.137 verabreichten Einzeldosen fielen sie leicht bis mäßig aus. Nur bei vier der 22 Kinder musste die Behandlung abgebrochen werden. Im Labor zeigte sich ein Anstieg von Erdnussspezifischem IgG4 und die Konzentration der Interleukine IL-5, IL-4 und IL-2 war danach vermindert.

Kommentar: Patienten mit schwerer Erdnussallergie müssen sich immer vor anaphylaktischen Reaktionen fürchten. Ein möglicher Schutz könnte eine orale Immunisierung bieten. Eine - zugegeben aufwendige - Hyposensibilisierung führt offensichtlich bei über der Hälfte von ih-
Kommentar: Waldorfschulen sind anthroposophisch geführte Schulen. Anthroposophen lehnen das Impfen ab, und anthroposophische Ärzte sind der Auffassung, dass Infektionskrankheiten wie Masern wichtige „Meilensteine“ in der Entwicklung eines Kindes seien. Die Kinder in Waldorfschulen bleiben daher häufig ungeimpft. So kommt es, dass sich Masernendemien ziemlich regelmäßig in solchen Institutionen ereignen.

Der letzte große Ausbruch von Masern in Deutschland war 2006. Damals erkrankten 2.300 Personen, 414 davon so schwer, dass sie ins Krankenhaus mussten. Zwei Patienten starben an Enzephalitis. Vor diesem Hintergrund klingt das Wort „Meilenstein“, wie ich finde, reichlich zynisch.

Prof. Dr. Ezard Ernst

Roggendorf $\mathrm{H}$ et al. Spotlight on measles 2010: measles outbreak in a mainly unvaccinated community in Essen, Germany, MarchJune 2010. Euro Surveill 2010; 15. pii: 19605

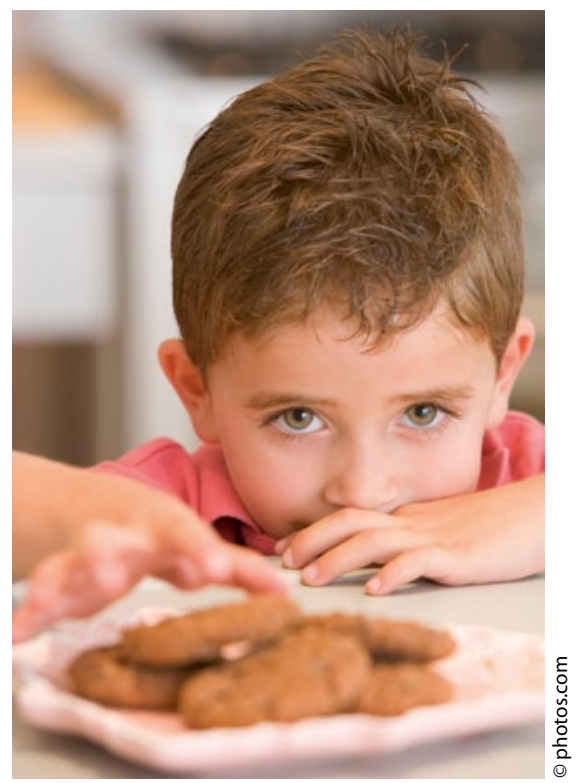

Nach einer Immuntherapie muss man keine Angst mehr vor Erdnussspuren in Lebensmitteln haben.

nen zur ausreichenden Toleranz gegen Erdnuss. Dr. Ulrich Mutschler

Blümchen $\mathrm{K}$ et al. Oral peanut immunotherapy in children with peanut anaphylaxis. J Allergy Clin Immunol 2010; 126: 83-91 